\title{
Experimental and Numerical Simulation of Wheel-Rail Adhesion and Wear Using a Scaled Roller Rig and a Real-Time Contact Code
}

\author{
Nicola Bosso and Nicolò Zampieri \\ Politecnico di Torino, DIMEAS, Corso Duca degli Abruzzi 24, 10129 Torino, Italy \\ Correspondence should be addressed to Nicola Bosso; nicola.bosso@polito.it
}

Received 9 April 2013; Accepted 10 August 2013; Published 23 February 2014

Academic Editor: Mehdi Ahmadian

Copyright (C) 2014 N. Bosso and N. Zampieri. This is an open access article distributed under the Creative Commons Attribution License, which permits unrestricted use, distribution, and reproduction in any medium, provided the original work is properly cited.

\begin{abstract}
The work shows the use of a scaled roller rig to validate a real time wheel-rail contact code developed to study the wheel rail adhesion and the wear evolution. The code allows the profiles to change at each time step in order to take into account the material loss due to the wear process. The contact code replicates a testing machine composed of a roller rig with a prototype of a single suspended wheelset pressed onto it with a variable load. The roller rig, developed at Politecnico di Torino, is used to validate and optimize the contact code referring to experimental data directly measured in real time. The test bench, in fact, allows measurement of specific kinematical quantities and forces that are elaborated by the real-time code in order to produce numerical results for comparison with the experimental ones. This approach can be applied both to the determination of wheel-rail adhesion and to the wear process. The test rig is also equipped with a laser profilometer that allows measurement of the wheel and rail profiles with a very high accuracy.
\end{abstract}

\section{Introduction}

The work deals with the use of a scaled roller rig used to perform wear and adhesion studies representative of the behaviour of a railway vehicle.

The aim of the work is to demonstrate how this approach can be a valid alternative with respect to other techniques, with the evident benefits of lower costs and reduction of the time required to perform the tests.

The proposed approach presents substantial aspects of interest as regards the state of the art, since simpler and less expensive tests can be performed, as already shown by the authors in [1].

The most common approach used to perform adhesion or wear tests on materials is the use of pin-on-disk $[2,3]$ or twin-disk systems. The concept of the twin-disk system is very similar to the use of a roller rig and can be used for adhesion studies [4] and for wear studies [5-7]. Most of the tests, carried out to define wear models or to determine the material properties, have been performed using this method. The twin-disk system is simple and efficient; it consists in the use of two cylindrical rollers pressed into contact and rotating with different peripheral speeds. The variation of the relative velocity and of the contact pressure allows performing the test under different conditions.

The tests performed with this system cannot be directly compared with a railway vehicle, since, contact geometry and slip conditions are different at the contact interface. Therefore, in order to simulate the effect of sliding and contact pressure occurring between wheel and rail, a numerical method is required, for this reason; only the material data can be directly obtained from the twin-disk tests. Under these conditions, the errors in the numerical model affect the predictions, and the test results cannot be used to validate the model. From the theoretical point of view, the optimal method to validate an adhesion or a wear model, representing a real railway vehicle, is the use of tests on the track. This method, however, requires a large investment in order to perform the tests due to track occupation, vehicle parameterization and instrumentation, use of a track geometry measurement vehicle to identify the track quality before the passage of the train, and all of these for a long mileage. Furthermore, the twin-disk method applies 
conveniently only in cases of wheel wear $[8,9]$ or adhesion studies $[10,11]$, where it is adopted especially to test antislip or antiskid systems.

An alternative to the test on the track consists of the use of a full scale roller rig. This approach, already adopted by Carter at the beginning of the 19th century to perform adhesion tests on locomotives [12], gives substantial benefits, since the tests are performed on a test stand in a laboratory environment, without the requirement for line occupation. The tests can be performed on real vehicles [13] or real wheelsets [14], and the instrumentation of the test rig allows the achievement of repeatable and reliable measurements.

However, the tests performed on full scale roller rigs are rather expensive in terms of time and direct costs. Therefore, the use of a scaled roller rig, already used by several authors to perform dynamic tests and other studies on railway vehicles [15], can be a useful alternative. This method can be considered as an intermediate solution between the twindisk system and the full scale roller rig. The benefits consist of a lower cost and a reduced time required to perform the tests while preserving the geometrical characteristics of the railway system. Higher complexity is required to obtain equivalent data to a real vehicle running on the track, since a similitude approach and inherent errors related to the use of rollers must be considered [16-18]. Therefore, this approach can be used for an initial validation of wheel-rail wear or adhesion models.

The aim of the work is to show how to design and to perform adhesion and wear tests using a scaled roller rig. An innovative method is used to generate the required creepage by using a trailing wheelset and two independent motorised rollers. This method allows evaluating the performance of the contact algorithm using a very simple mechanical configuration (a single wheelset without any traction/braking system installed).

The use of a real-time system and the test rig allows testing the wheel-roller contact code and the wear model, developed by the authors [1], by using the measured data. The data measured by sensors are acquired by the real-time system that incorporates the data acquisition boards and a computer with installing the Labview-RT Real-Time operative system. Those data are used by the RTCONTACT contact code [19] to evaluate the friction forces and the material removed by the wear phenomenon. This phenomenon, in fact, needs to be studied during its evolution since the shape of the profile changes over time, thus affecting the contact pressure, the curvatures, and the contact geometry in general. The use of a real-time contact model is also suitable to evaluate wheel-rail adhesion, as it is possible to calculate, with a good accuracy, the friction force acting on the contact area. By using the realtime architecture, it is also possible to compare the numerical results and the experimental ones, directly during the test. In this way, the numerical model is able to operate directly using the measured data.

\section{Description of the Test Rig}

The test rig is composed of a suspended wheelset supported by a couple of independent rollers. Each roller is connected to an electric motor that can be driven independently, in order to generate a creepage at the contact interface between the wheel and the roller. The wheelset is not connected to any traction system; therefore, the creepages at the left and right contact areas only depend on the velocity of the rollers and on the friction forces equilibrium.

When different values of velocity, for example, $\omega_{1}>\omega_{2}$, are given to the two rollers, the velocity of the wheelset $\omega_{3}$ becomes

$$
\omega_{1}>\omega_{3}>\omega_{2} .
$$

As a consequence, the following contributions to the longitudinal creepages are obtained on the two contact patches:

$$
\xi_{\omega_{1}}=\frac{\omega_{1} R_{1}-\omega_{3} R_{3}}{\omega_{1} R_{1}}, \quad \xi_{\omega_{2}}=\frac{\omega_{2} R_{2}-\omega_{3} R_{3}}{\omega_{2} R_{2}} .
$$

Equation (2) is valid with not vanishing $\omega_{1}$ and $\omega_{2}$. It represents the most important term of the longitudinal creepage, when the difference between the velocities of the two rollers is representative of a traction or braking condition. From (2) it is evident that the two contact patches have opposite creepages. Figure 1 shows the concept of the roller rig as used in this work.

This control strategy of the roller rig is not common in the literature; in fact, this solution is not realistic with respect to a real vehicle during traction/braking operation, where both wheels have the same sign of the creepage. A similar situation only occurs on curves, where the velocities on the inner and outer wheel are different.

However, this control strategy has not been adopted in this work to reproduce curving behaviour, but for a different reason. In fact, it allows testing the performances of the contact code on a roller rig with the simplest procedure and mechanical system. No traction or braking system is required on the wheelset. Nevertheless, information concerning the behaviour under traction/braking conditions and two different creepage values can be obtained simultaneously. The left/right creepage value can be modified by acting on the normal load of each wheel or on the friction coefficient. It must be observed that it is not easy to keep a constant ratio between the two creepages, due to the wheelset movement and local variations in the contact conditions. This effect is not a big problem because it is possible to measure accurately the angular velocity of the rollers and the velocities and displacements of the wheelset, in order to assign the proper creepage to each contact, during the data acquisition.

2.1. Mechanical Arrangement. The mechanical system (see Figure 2) is composed of two rollers, each one supported by high precision angular contact bearings, secured in two bearing boxes. The rollers have interchangeable rings, where the $1: 20$ canted rail profile is reproduced in 1:5 scale. The rail profile has been modified with respect to the standard European UIC60 rail profile, in order to compensate the curvature of the roller and to obtain the same $a / b$ ratio of the contact area on the tread of a wheelset on a straight track, as shown in [17]. 


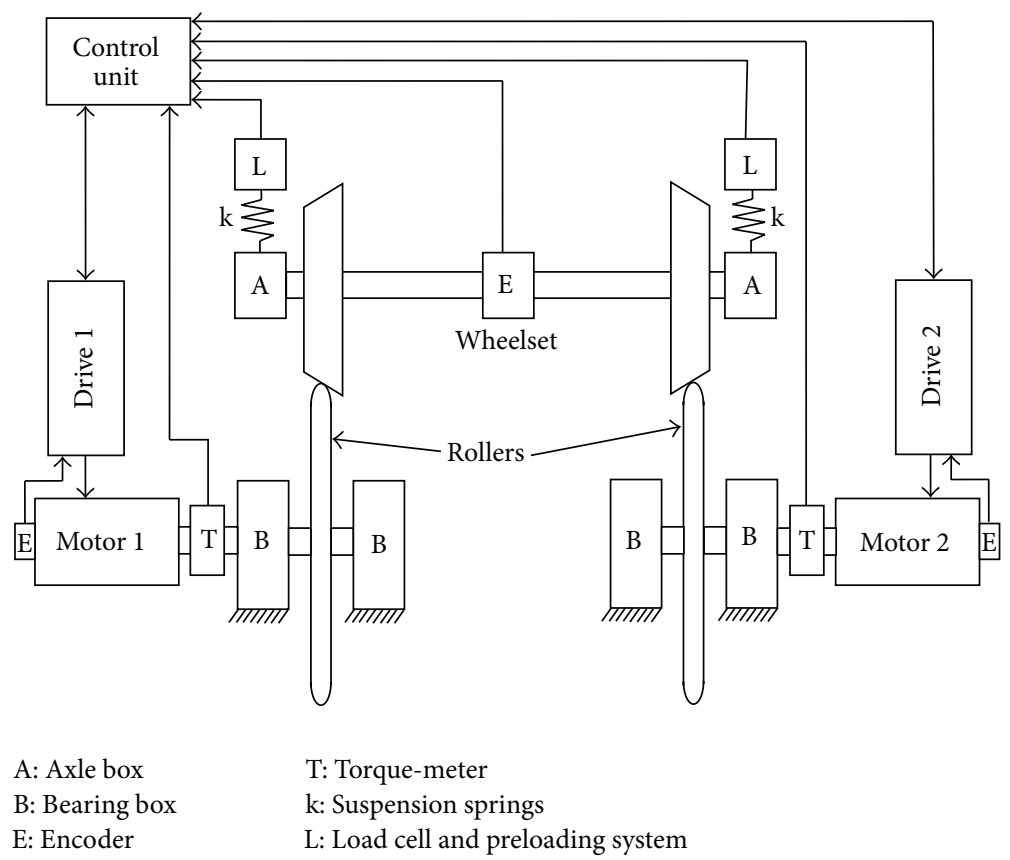

FIGURE 1: Schematic of the roller rig with a single suspended wheelset.

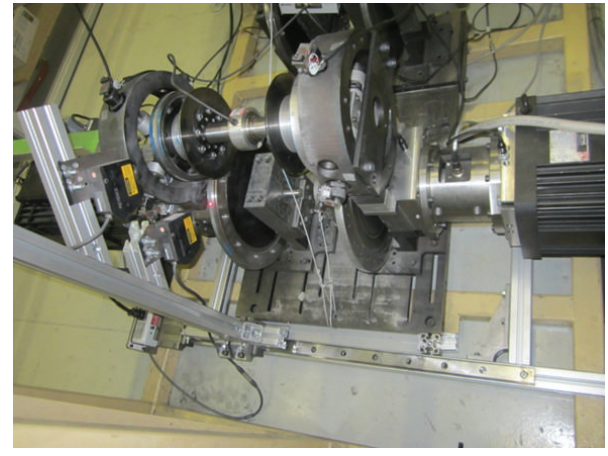

Figure 2: Roller rig with single suspended wheelset.

Each roller is connected to a permanent magnet synchronous motor, by means of a torque transducer used to measure the applied torque. The motors are equipped with high resolution (65536 pulses per round) encoders to measure the angular velocity and give a feedback to the motor control. The wheelset is also equipped with interchangeable profiles, where conical or shaped profiles can be used. It has been designed in 1:5 scale according to the similitude approach proposed by Jaschinski et al. [15] and Bosso et al. [17]. The most important geometrical and inertial data are shown in Table 1.

The angular velocity of the wheelset is measured using an encoder mounted centrally on the wheelset axle. The wheelset is elastically connected to the base of the test rig with a structure including the springs and an appropriately designed preload system that allows the load regulation and its measure using strain-gauge sensors. Each axle box is connected to the structure using two longitudinal helical
TABLE 1: Wheelset geometrical and inertial data.

\begin{tabular}{lc}
\hline \multicolumn{2}{c}{ Wheelset properties $(1: 5$ scale $)$} \\
\hline Nominal roller radius & $0.185 \mathrm{~m}$ \\
Nominal wheel radius & $0.0944 \mathrm{~m}$ \\
Mass & $13 \mathrm{~kg}$ \\
Moment of inertia $I_{x}$ & $0.28 \mathrm{Kg} * \mathrm{~m}^{2}$ \\
Moment of inertia $I_{y}$ & $0.035 \mathrm{Kg} * \mathrm{~m}^{2}$ \\
Moment of inertia $I_{z}$ & $0.28 \mathrm{Kg} * \mathrm{~m}^{2}$ \\
\hline
\end{tabular}

springs, one lateral spring and a vertical spring, in order to be able to independently modify the stiffness and the load in each direction. The test rig is also equipped with a profilometer based on laser sensors with very high resolution $(0.1 \mu \mathrm{m})$, designed with one degree of freedom in the lateral direction by means of a linear guide with preloaded ball recirculation pads. This device, shown in Figure 2, is able to measure the profiles of the rollers and of the wheelset during the tests.

2.2. Control and Data Acquisition. The test rig is equipped with a real-time (RT) system which is responsible for the control of the rig, the data acquisition, and the simulations of the wheel-rail/roller contact in order to compute the wheel and roller wear and adhesion. The architecture of the RT system is an industrial PC developed by National Instruments (NI-PXI 8110) based on an Intel Core 2 Quad Q9100 2.26 GHz processor with the operative system LabVIEW-RT. This system is connected with a PXI-SCXI acquisition system with different I/O cards:

(i) PXI4472B (ICP accelerometers and filtered voltage), used to measure accelerations and displacements, 


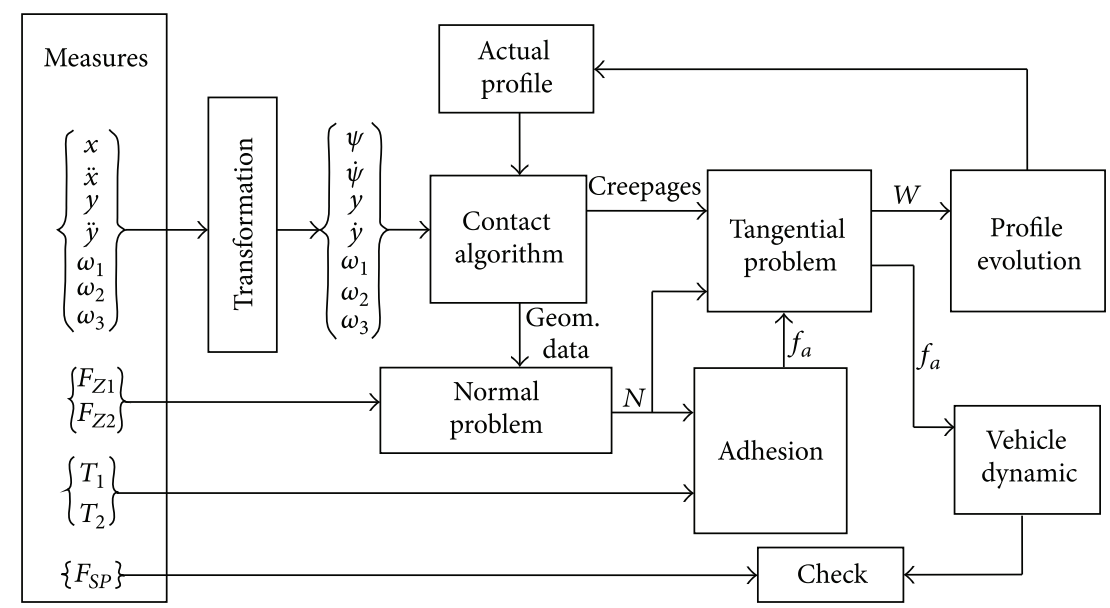

FIGURE 3: Scheme of the tasks performed by the control/acquisition system.

(ii) SCXI 1314 (strain gauges), used to measure the forces on the springs,

(iii) PXI 7330 (encoder input), used to measure the angular velocities,

(iv) SCXI 1308 (current input), used to communicate with the drives,

(v) PXI 6259 (analog output), used to communicate with the drives.

This control system communicates with the drives of the electric motors using current I/O analog signals with 16 bit resolution. The signals have been divided into three channels to reduce the noise, as shown in [1]. The drives are equipped with a programmable unit, where specific codes for a torque control and a velocity control application are implemented. The motors are controlled using a PI current loop (proportional gain 128 and integrative gain 226) and a PID velocity loop (proportional gain 0.45 , integrative gain 1.5 , and derivative gain 0.02 ).

The roller rig can be used to perform adhesion or wear tests. In the case of wear tests, a velocity control is used to impose different velocities to the two rollers. In the case of adhesion tests, since the adhesion curve must be determined, the creepage has to be changed during the test. This operation can be made in both torque and velocity control. Figure 3 shows the process of simulation and data acquisition performed by the RT-system in the case of wear tests. During the adhesion tests, the blocks relative to wear calculation and profile evolution are not required, since the output of the test is the traction coefficient $f_{a}$ versus the creepage.

The measured data, including displacements or accelerations of the wheelset, are used to estimate the full set of creepages and the contact point location on wheel and roller. The RT algorithm includes a model of the suspended wheelset used to simulate the forces at the suspension elements for the longitudinal direction. Those simulated forces $F_{\mathrm{SP}}$ are also measured, using load cells located on the suspension connections and visible on Figure 2, and compared with the simulated values to validate the model. The main purpose of the RT contact model is to simulate the profile wear during the test. The wheel and roller profiles, starting from the measured values, are updated at each step of the simulation (20 ms). The laser profilometer is used to perform, at discrete intervals, as shown in Table 6 , a new physical measure of the profiles that are then stored in the code and compared with the simulated ones. This process allows the wear coefficients to be refined and to obtain accurate results at the end.

The wheelset displacements, measured with laser sensors, can be replaced by double integration of the measured accelerations, performed with ICP accelerometers.

This second approach, still under investigation, can be used to adopt this method on a real vehicle, where it is not simple to obtain the displacement measures.

\section{RT Algorithm}

The contact algorithm used in this work is called RTCONTACT [19] and it is based on a quasielastic contact model. The code is written in $\mathrm{C}++$ code and compiled into dynamic link libraries (DLLs). The DLLs are integrated in the LabVIEWRT project using the "Call Library Function Node" block. The advantage of using the DLLs is that the contact code can be executed in the same environment of the acquisition, avoiding to export the acquired data outside LabVIEW. The code is developed in order to minimize the calculation time, allowing the execution of the code in a real-time environment. The nonlinearities, due to the contact, result in high computational loads for the CPU, but the RTCONTACT code, because of its computational efficiency (more than 10000 iterations per second on an AMD Opteron single core processor [19]), is able to work in real time; therefore it is suitable for use on control systems. The whole algorithm is built using a modular approach (see Figure 3), which allows the parallel execution of the different modules on different cores. In the present work, the algorithm has been parallelized on the four cores of an Intel Core 2 Quad Q9100 2.26 GHz processor. 
3.1. Contact Algorithm. The code is developed using the dynamic management of the memory, to minimize the amount of memory used by the contact code during the execution. This characteristic of the code makes the algorithm suitable to be used in embedded applications that often have a small amount of memory available. The contact algorithm can be divided into four main modules as shown in Figure 3.

(i) The Geometrical Module. The aim of this module is the evaluation of the contact points position and the calculation of the most important geometrical quantities in the contact patches (the contact angle $\gamma$, the rolling radii of the wheel and the rollers $r_{w}$ and $r_{r}$, the transverse curvature radii of the wheel, and the rollers $R_{w}$ and $R_{r}$ ). The inputs to this module are the lateral position of the wheelset $y$ and the yaw rotation $\Psi$ that are measured on the axle boxes of the wheelset using laser displacement sensors (resolution $0.1 \mathrm{um}$ ). In this case, the effect of the yaw angle $\Psi$ on the contact point position (e.g., the contact point shift) is small, due to the high stiffness used on the longitudinal springs. This aspect is important to reduce the effects on the yaw angle due to the particular method used to apply the traction torque to the wheelset. The geometrical algorithm is the part of the contact code that requires the highest calculation time (about $80 \%$ of the calculation time to execute the whole algorithm). This module is based on the pseudoelastic contact theory, and it considers the case of two-dimensional geometry, where the cross-sections of the wheel and the roller profiles are used to find the contact point position. The quasielastic contact allows a penetration between the two profiles, and its value $\varepsilon$ is chosen to be very small and less than $10^{-12} \mathrm{~m}$, which substantially corresponds to a rigid contact.

(ii) The Hertz Module. In this section the semiaxes of the contact areas are calculated according to the Hertz theory. In order to be able to use the Hertz theory, when friction between the contact surfaces occurs, the quasiidentity hypothesis is adopted. This hypothesis states that the tangential pressures, due to friction, do not affect the shape of the contact area. The inputs of this module are the curvature radii of the wheel and the roller in the contact patch (these quantities are calculated in the geometrical module using the Frenet formulas) and the normal load.

(iii) The kinematical Module. In this module the kinematical creepages between the wheel and the roller are calculated. These quantities are defined by Kalker in [20] as the ratio between the relative velocity and the reference velocity. According to Kalker, three different creepages can be defined: the longitudinal creepage $\xi$, the lateral creepage $\eta$, and the spin creepage $\Phi$ as follows:

$$
\xi=\frac{\Delta V_{x}}{V_{0}}, \quad \eta=\frac{\Delta V_{y}}{V_{0}}, \quad \Phi=\frac{\Delta \omega_{N}}{V_{0}} .
$$

In the experimental tests shown in this paper the lateral creepage and the spin creepage are very small, because the lateral velocity and the yaw angle are very small on tangent track. Thus, the longitudinal creepage is more important as this quantity significantly affects the wear regime. Its most important contribution is given by (2), while the complete formulation for a roller rig can be found in [18]. The inputs of the kinematical module are the actual rolling radii of the wheel $r_{w}$ and the roller $r_{r}$ (calculated in the geometrical module), the wheelset velocities $\{\dot{q}\}$, and displacements $\{q\}$.

(iv) The Tangential Force Calculation Module. In this module the friction forces $F_{x}$ and $F_{y}$ are calculated by using the FASTSIM algorithm [21] developed by Kalker. The inputs of this module are the semiaxes $a, b$ of the contact ellipse; Kalker's coefficients $C_{x}, C_{y}$, and $C_{z}$, which are interpolated from a look-up table; the creepages $\xi$, $\eta$, and $\Phi$; the friction coefficient $\mu$; and the normal load $N$. This module is computed in the "Tangential Problem" block in Figure 3 because the contact forces are calculated simultaneously to the wear distribution.

3.2. Normal Problem. The normal forces are determined using both the measured data and the results of the geometrical module. As may be seen in Figure 1, the vertical forces on the axle boxes are measured using two vertical load cells. The normal forces are calculated from the dynamic equilibrium of the wheelset, considering the wheel profile and the inertial effects, as shown in

$$
\begin{aligned}
& N_{1}=\frac{F_{z 1}+F_{z 2}+m_{w}\left(\ddot{y} \cot \left(\gamma_{2}\right)-g-\ddot{z}\right)}{\sin \left(\gamma_{1}\right) \cot \left(\gamma_{2}\right)-\cos \left(\gamma_{1}\right)}, \\
& N_{2}=\frac{F_{z 1}+F_{z 2}+m_{w}\left(\ddot{y} \cot \left(\gamma_{1}\right)-g-\ddot{z}\right)}{\sin \left(\gamma_{2}\right) \cot \left(\gamma_{1}\right)-\cos \left(\gamma_{2}\right)} .
\end{aligned}
$$

The lateral friction forces can be neglected in equilibrium since the lateral creepages are not important in this case. The accelerations $\ddot{z}$ and $\ddot{y}$ are measured using the accelerometers. It is evident that, in order to evaluate correctly the normal load, it is necessary to have a contact algorithm that can identify the contact point because the contact angles $\gamma_{1}$ and $\gamma_{2}$ are determined in the geometrical module.

3.3. Adhesion Estimation. The aim of the adhesion tests is the estimation of the force-creepage relation. The 1:5 scaled test rig is a single suspended wheelset, and the motion is applied by two rollers that are independently driven, as shown in Figure 1. Motor 1 is controlled to keep a constant velocity, while motor 2 can be controlled both in velocity and in torque. Before beginning the experimental test, the two motors run at the same velocity but, as the test starts, in the first case the velocity of motor 2 is reduced in order to increase the creepage, while, in the second case, the resistant torque on motor 2 is gradually increased. The first method allows the determination of the creepage curve for high values of creepage, while the second allows investigation of the creepage curve close to the initial (linear) part. The code is divided into two main parts: the acquisition module, which acquires the data from the real system, and the torque calculation module, which controls motor 2 . The longitudinal friction force, acting on the contact point, can be calculated 
TABLE 2: Values of the wear coefficient according to [22].

\begin{tabular}{lccc}
\hline Wear coefficient $K$ & $v_{\text {slip }}<0.2 \mathrm{~m} / \mathrm{s}$ & $0.2 \mathrm{~m} / \mathrm{s}<v_{\text {slip }}<0.7 \mathrm{~m} / \mathrm{s}$ & $v_{\text {slip }}>0.7 \mathrm{~m} / \mathrm{s}$ \\
\hline$P>0.8 * \mathrm{H} \mathrm{MPa}$ & & $K_{1}=300-400 \times 10^{-4} \mathrm{~m} / \mathrm{km}$ & \\
$P<0.8 * \mathrm{H} \mathrm{MPa}$ & $K_{2}=1-10 \times 10^{-4} \mathrm{~m} / \mathrm{km}$ & $K_{3}=30-40 \times 10^{-4} \mathrm{~m} / \mathrm{km}$ & $K_{4}=1-10 \times 10^{-4} \mathrm{~m} / \mathrm{km}$ \\
\hline
\end{tabular}

TABLE 3: Values of the wear coefficient used in this work: the coefficient $K$ is expressed in $\mathrm{m} / \mathrm{km}$ rolled.

\begin{tabular}{|c|c|c|c|c|c|c|c|}
\hline \multirow{2}{*}{ Wear coefficient $K$} & \multicolumn{7}{|c|}{$v_{\text {slip }}$} \\
\hline & $<0.1$ & $0.1 \div 0.2$ & $0.2 \div 0.3$ & $0.3 \div 0.4$ & $0.4 \div 0.5$ & $0.5 \div 0.7$ & $>0.7$ \\
\hline \multicolumn{8}{|l|}{ Wheel } \\
\hline$P>0.8 * \mathrm{H} \mathrm{MPa}$ & & & & $370 \times 10^{-4}$ & & & \\
\hline$P<0.8 * \mathrm{H} \mathrm{MPa}$ & $1 \times 10^{-4}$ & $3.6 \times 10^{-4}$ & $8.5 \times 10^{-4}$ & $29 \times 10^{-4}$ & $31.8 \times 10^{-4}$ & $18 \times 10^{-4}$ & $1 \times 10^{-4}$ \\
\hline \multicolumn{8}{|l|}{ Roller } \\
\hline$P>0.8 * \mathrm{H} \mathrm{MPa}$ & & & & $520 \times 10^{-4}$ & & & \\
\hline$P<0.8 * \mathrm{H} \mathrm{MPa}$ & $2 \times 10^{-4}$ & $7.8 \times 10^{-4}$ & $13.3 \times 10^{-4}$ & $48.2 \times 10^{-4}$ & $53 \times 10^{-4}$ & $21.7 \times 10^{-4}$ & $2 \times 10^{-4}$ \\
\hline
\end{tabular}

from the measured torques on motor 1 and motor 2 using (5).

$$
F_{1}=\frac{T_{1}}{r_{r 1}}, \quad F_{2}=\frac{T_{2}}{r_{r 2}} .
$$

In (5), $r_{r 1}$ and $r_{r 2}$ are the rolling radii of the rollers and they are calculated by the contact model. The creepages are calculated using the velocities measured on the rollers and on the wheelset according to (2). Observing (5), for the creepages calculation, it is also necessary to know the rolling radii of the wheels and the rollers. These quantities are provided by the geometrical module of the RTCTONTACT code, as function of the lateral position and the yaw angle of the wheelset (Figure 3), measured with the laser sensors.

3.4. Tangential Problem and Wear Calculation. The amount of material removed due to the wear process is calculated in a modified FASTSIM algorithm. The original Kalker's algorithm has been modified in order to evaluate the material loss. The method, adopted to calculate the material removed from the wheel and the roller, is based on the Archard's theory. This model has been used by many researchers in the field. It is usually used in the tribology field for modeling sliding wear. Archard's wear model $[22,23]$ considers that the volume of material worn away $V$ is proportional to the sliding distance $S$ and the normal force $N$ and inversely proportional to the Brinell hardness $H$ of the worn material, as shown in

$$
V=\frac{K S N}{H} \text {. }
$$

According to [22], the wear coefficient $K$ can be determined from a wear map (shown in Table 2) for relevant material combinations at dry conditions. The most important regimes for railway operations are the $K_{2}$ and $K_{3}$ areas, although both higher pressures and larger slip may occur in narrow curves or at very poor adhesion conditions, respectively.

In this work the wear coefficient map has been calculated according to the experimental data, and the map has been divided into more areas with respect to Table 2 . In the first
TABLE 4: Values of the Brinell hardness of the wheel and the roller.

\begin{tabular}{lc}
\hline & Hardness HB (MPa) \\
\hline Wheel (Fe360) & 100 \\
Roller (Fe510) & 160 \\
\hline
\end{tabular}

phase of the work the profiles have been worn at different creepage levels and loads in order to determine the wear coefficients. Also during the tests shown in this work, the wear coefficient map has been modified so as to tune the wear model with the experimental data. The final wear map used in this work is shown in Table 3.

The tests, shown in this work, have been made using softer wheel and roller material with respect to those used in [22]. The materials are of course not a realistic representation of the real wheel-rail interaction, but they allow the tests to be performed faster without any implication regarding the development of the contact algorithm, especially during the first phases, when several tests are required to tune and correct the algorithm. The construction of the wheels and, especially, the rollers in proper materials is rather complex and expensive since real materials are supplied in the form of rails and not of rounds or plate of adequate dimensions to obtain a roller to be fitted on the roller rig. The correction of the algorithm, once has been tested and validated, can be easily done by performing a final test using the proper material, but this activity is postponed to a future phase of the research.

Table 4 shows the hardness of the materials used to construct the wheels and the rollers.

In order to evaluate the tangential pressure distribution on the contact area, the FASTSIM algorithm discretizes the contact region along both the $x$ and the $y$ direction. In this work, the number of elements $C$ along the two directions is the same and equal to 20 . For this reason the total number of elements on the contact area is 400 . If we apply (6) to a single element of the contact area we obtain

$$
V=\frac{K S_{e} N_{e}}{H}
$$


The subscript index $e$ shown in (7) is used to indicate that the equation is relative to a single element of the contact area. Dividing both sides of (7) by the area of the single element $(d x \times d y)$ we obtain the wear depth at the center of the element $U_{e}$ as shown in

$$
U_{e}=\frac{K S_{e} p_{e}}{H} .
$$

The sliding distance $S_{e}$ on the element can be calculated using (9), where $d x$ is the length of the element along the $x$ direction, as follows:

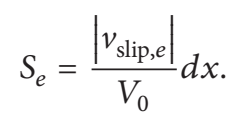

In (9), the slip velocity on the element $v_{\text {slip }, e}$ is calculated according to

$$
v_{\text {slip }, e}=K_{v} \sqrt{s_{x, e}^{2}+s_{y, e}^{2}} \text {. }
$$

In (10), $s_{x, e}$ and $s_{y, e}$ are the dimensionless velocities defined by Kalker in the FASTSIM algorithm. The constant $K_{v}$ is used to convert Kalker's dimensionless velocities into the real velocities. The constant $K_{v}$ can be calculated using (11), where $a$ and $b$ are the semiaxes of the contact ellipse and $N$ is the normal load on the contact area, as follows:

$$
K_{v}=\frac{2 N \mu V_{0} L_{\mathrm{eq}}}{\pi a^{2} b} .
$$

The flexibility equivalent parameter $L_{\text {eq }}$ can be calculated as the weighted mean of $L_{1}, L_{2}$, and $L_{3}$, as suggested by Kalker in [21] and shown in (12). In the equation $\xi, \eta$, and $\Phi$ are the longitudinal, lateral, and spin creepages, respectively:

$$
L_{\mathrm{eq}}=\frac{L_{1}|\xi|+L_{2}|\eta|+L_{3}|\Phi| \sqrt{a b}}{\sqrt{\xi^{2}+\eta^{2}+a b \Phi^{2}}} .
$$

The variable $V_{0}$ in (11) is the reference velocity that can be simply calculated as the product of the roller velocity $\omega_{r}$ and the roller rolling radius $r_{r}$, as shown in

$$
V_{0}=\omega_{r} r_{r}
$$

The normal pressure $p_{e}$ acting on the element of the contact area can be calculated by using the dimensionless pressure $z$, as computed by Kalker in the FASTSIM algorithm and shown in

$$
p_{e}=K_{p} z
$$

The constant $K_{p}$ is calculated according to

$$
K_{p}=\frac{3 N}{2 \pi a b} .
$$

After the wear depth has been calculated on each element, the mean value on each strip along the $x$ direction is calculated. In this way, it is possible to build a wear curve that is only a function of the $y$ coordinate on the contact plane, so the variation range of the function is $[-b, b]$. This process is necessary since the contact model considers two-dimensional profiles, and so the profiles are only a function of the lateral $y$ and vertical $z$ coordinates. Obviously the wear depth is calculated only in the region of the contact area where slip occurs, while in the adhesion region there is no material removal and the wear depth is therefore zero.

The mean value of the wear along the $x$ strip is calculated according to

$$
U_{x}(y)=\frac{\sum_{i=1}^{C} U_{e, i}}{C} .
$$

The wear depth calculated according to (16) is uniformly distributed along the circumferential direction of the wheel and the roller. Obviously the algorithm calculates the $U_{x}(y)$ distributions for the wheel and the roller, which are different since the material and the rolling radii of the two bodies are not the same. The distributions along the circumferential direction for the wheel $U_{S}^{w}(y)$ and the roller $U_{S}^{r}(y)$ are calculated according to

$$
\begin{gathered}
U_{S}^{r}(y)=\frac{U_{x}^{r}(y)}{2 \pi r_{r}} \omega_{r} r_{r} \Delta t, \\
U_{S}^{w}(y)=\frac{U_{x}^{w}(y)}{2 \pi r_{w}} \omega_{w} r_{w} \Delta t .
\end{gathered}
$$

In (17), the subscript $r$ is used for the roller and the subscript $w$ for the wheel. Obviously the wear damage is calculated both on the right and the left side. The variable $\Delta t$ is the amount of time that elapses between two calculations of the wear depth. This calculation is performed in the RT algorithm in $20 \mathrm{~ms}$. The wear distributions are calculated in the module called "Tangential Problem" in Figure 3, and these are used to evaluate the profile evolution over time. The last operation is performed in the module called "Profile Evolution."

3.5. Profile Evolution. In order to study the evolution of the wear over time, it is necessary to modify the profile of the wheels and the rollers at each time step. This operation is very important since the material loss due to wear modifies the shape of the profiles and, as a consequence, the contact point position and the contact angle are affected by the actual shape of the profile. For this reason, in order to compare the numerical results and the experimental data, it is necessary to develop a contact algorithm able to evaluate the wear trend and use this data to update the profiles. The wear depth on the wheel and on the roller is calculated in the "Tangential Problem" block, and this data is used to update the profiles. The wear depth is calculated on the contact area and so it is perpendicular to the plane tangential to the contact point. Therefore, to remove the material from the profile, it is necessary to rototranslate the wear curve on the profile. The material is removed from the profile at each iteration and, after 10 iterations, a filter (sliding mean) is used to regularize the profile of the wheel and the roller.

The first operation to update the profile is the determination of the section of the wheel-roller profile affected by 


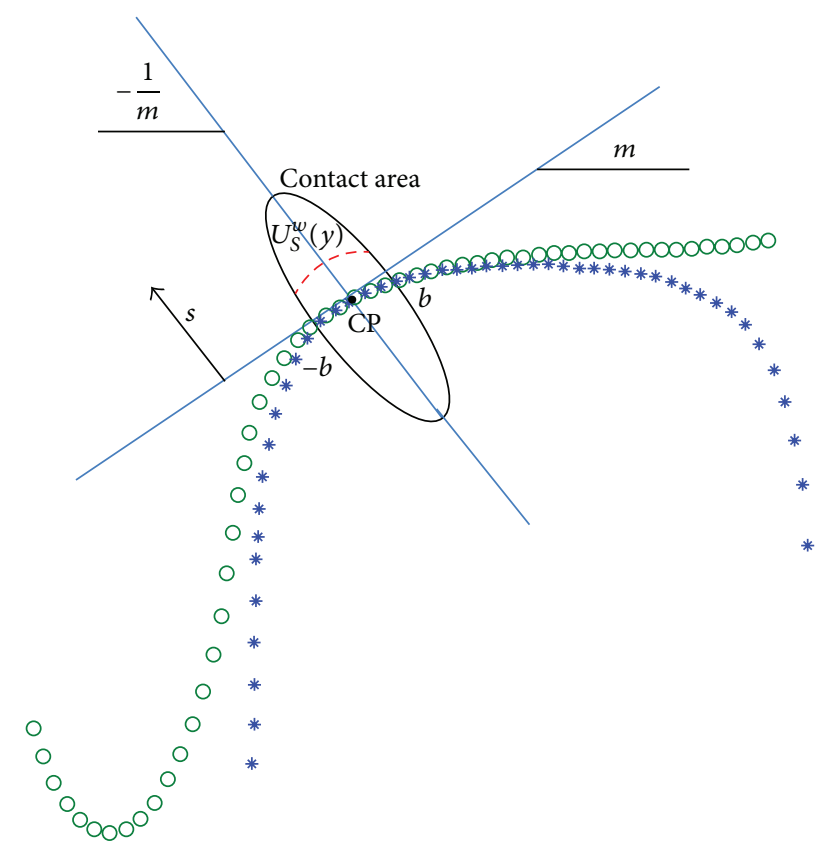

FIGURE 4: Method adopted to remove the material from the profiles.

the wear phenomenon (see Figure 4). Equation (18) shows the range of the profile where there is loss of material, depending on the $y$-coordinate of the contact point $y_{C}$, the contact angle $\gamma$, and the semiaxis of the contact ellipse $b$, as follows:

$$
\left[y_{C}-b \cos (\gamma) ; y_{C}+b \cos (\gamma)\right]
$$

Region of the profile affected by wear.

The function of the wear depth $U_{S}(y)$ is perpendicular to the profile in the contact region and, for this reason, it is necessary to parameterize the line perpendicular to the profile and passing through the point of the profile, included in the range shown in (18).

The line is parameterized as a function of the normal distance from the profile $s$, as shown in Figure 4. Equation (19) shows the relationship of the line parameterized as a function of $s$ in the absolute system:

$$
y_{\perp}=\frac{s}{\sqrt{1+m^{2}}}, \quad z_{\perp}=\frac{m s}{\sqrt{1+m^{2}}} .
$$

In (19), the parameter $m$ is the angular coefficient of the line tangent to the profile and, for this reason, at each time step it is necessary to interpolate the profile in order to evaluate the first derivative. This operation is performed in the geometrical module, during the calculation of the contact point. The function $U_{S}(y)$ is reinterpolated and discretized on the basis of the same number of points of the wheel-roller profiles laying in the range of (18). A discrete function $U_{S, j}$ is obtained, and it represents the wear along the normal line passing through the $j$ point of the considered profile.
Substituting in (19) the variable $s$ with the wear depth, it is possible to calculate the "wear profile" to be removed from the wheel-rail profiles, as shown in

$$
y_{\text {wear }, j}=\frac{U_{S, j}}{\sqrt{1+m^{2}}}, \quad z_{\text {wear }, j}=\frac{m U_{S, j}}{\sqrt{1+m^{2}}} .
$$

The worn profiles become

$$
\begin{aligned}
& y p_{i+1, j}=y p_{i, j}-y_{\text {wear }, j}, \\
& z p_{i+1, j}=z p_{i, j}-z_{\text {wear }, j} .
\end{aligned}
$$

In (21), $y p_{i, j}$ and $z p_{i, j}$ are the coordinates of the wheel or the roller profile at the $i$ th iteration at point $j$. The same equations can, in fact, be used both for the roller and the wheel profiles, obviously considering different values of $m$ and $U_{s, j}$. The points of the profiles that are modified according to (21) are only the points of the profile whose lateral coordinate is included in the range shown in (18).

When the program starts, the code allocates the memory locations to store the wheel and the roller profiles and loads the initial profiles provided by the user by using a text file. After this operation, the code modifies the profiles at each time step, in the region where the wear occurs, and overwrites the existing profiles. During the tests the profiles are saved by the code into text files every $2000 \mathrm{~m}$, in order to evaluate the wear process over time. As mentioned earlier, before starting a new test, the wheel and the roller profiles are measured by using laser sensors and compared with the profiles calculated during the simulation. In this way, it is possible to modify and tune the wear coefficients in order to characterize the material used to build the wheels and the rails.

The process to refine the wear coefficients at the moment is not automatic but consists in a manual procedure: after the test, the numerical and experimental results are compared and the differences of worn material are computed. The code provides the statistical occurrences of different slip velocity values during the test. It has been assumed to reduce or increase the wear coefficients at each slip velocity in proportion to its relative occurrence.

The new test starts using the latest acquired profiles, in order to have a good accordance between the numerical and experimental data.

\section{Test Procedure}

The test procedure is different for the adhesion and the wear test. The adhesion test is performed starting with the two rollers running at the same velocity; then the velocity of the second roller is decreased. The reduction can be obtained by imposing a linear variation of the second roller velocity versus time. Another possibility is to impose on the second motor an increasing resistant torque, also according to a linear relationship versus time. The first approach gives better results for high creepage values; the second allows obtaining a better description of the curve close to the initial linear behaviour. The adhesion tests have been performed using roller profiles designed according to the UIC60 rail profile, canted at 1:20 and scaled at 1:5 with the methodology 
TABLE 5: Tests performed to evaluate the wear on the wheels and the rollers.

\begin{tabular}{lccccc}
\hline Test & Creep & Reference speed $(\mathrm{rpm})$ & Vertical load $(\mathrm{kg})$ & $\mathrm{km}$ travelled $(\mathrm{km})$ & Test time $(\mathrm{min})$ \\
\hline 1 & 0.25 & 500 & 53 & 21.8 & 17 \\
2 \\
2 & 0.05 & 500 & 50 & 23.1 & 29 \\
3 & 0.05 & 500 & 50 & 35.47 & 40 \\
4 & 0.1 & 500 & 48 & 10.2 & 61 \\
5 & 0.15 & 250 & 43 & & 35 \\
\hline
\end{tabular}

TABLE 6: Adhesion tests performed and results.

\begin{tabular}{lcccccc}
\hline Test & $\begin{array}{c}\text { Reference speed } \\
(\mathrm{rpm})\end{array}$ & $\begin{array}{c}\text { Wheel vertical load } \\
(\mathrm{kg})\end{array}$ & $\begin{array}{c}\text { Maximum adhesion } \\
\text { coefficient }\end{array}$ & $\begin{array}{c}\text { Steady adhesion } \\
\text { coefficient }\end{array}$ & $\begin{array}{c}\text { Initial slope } \\
(N)\end{array}$ & $\begin{array}{c}\text { Condition } \\
1\end{array}$ \\
1000 & 37 & 0.441 & 0.353 & 8700 & Dry \\
2 & 1000 & 37 & 0.220 & 0.212 & 8380 & Wet \\
3 & 700 & 37 & 0.435 & 0.342 & 7960 & Dry \\
4 & 700 & 37 & 0.242 & 0.220 & 7665 & Wet \\
5 & 358 & 42 & 0.461 & 0.402 & 12100 & Dry \\
6 & 358 & 42 & 0.315 & 0.282 & 12038 & Wet \\
7 & 358 & 47 & 0.488 & 0.403 & 18000 & Dry \\
8 & 358 & 47 & 0.293 & 0.278 & Wet \\
\hline
\end{tabular}

described in [17]. The roller profiles used for the adhesion tests have been built using C45 quenched and tempered, with a surface hardness of $300 \mathrm{HB}$. The wheel profiles have been built according to the $\mathrm{S} 1002$ profile, using a $39 \mathrm{NiCrMo} 3$ steel with a surface hardness of $240 \mathrm{HB}$.

The wear tests have been performed starting with the two rollers running at the same angular velocity; then the velocity of the second roller is reduced to a fixed value, kept constant during the remaining of the test. The motors are controlled using two velocity controls in electronic gearing mode (the ratio between the two velocities is kept constant). The wear tests, shown in this paper, have been performed using soft material for wheels and rollers, in order to accelerate the material removal. This choice has been adopted because, in this phase, the interest of the authors is to refine the wear model and to test the procedure. The setup of this process requires a large amount of wear to verify the capability of the model, and a softer material accelerates the operation.

In this work, conical wheel profiles are used to investigate wear, since, according to [23], the wear rate is different on the tread with respect to the gauge/flanging contact; the results are at the moment only representative for the tread. For the wear tests, the wheel has a conicity of 0,036 rad, and it is built using a Fe360 steel with a surface hardness of $100 \mathrm{HB}$. The rollers have the same profile used for the adhesion test, but they are built using a Fe510 steel with a surface hardness of $160 \mathrm{HB}$. The wear test plan and the parameters that have been used are shown in Table 5.

\section{Results}

This section discusses the results relative to the adhesion and the wear tests.
5.1. Adhesion Curve. As regards the adhesion tests, they are performed in different operating conditions, in order to estimate the longitudinal friction force versus the creepage. Different loads and dry/wet conditions are considered. The wet conditions are simulated by spraying a water jet from a nozzle onto the contact patch. Another parameter that affects the creepage curve is the reference speed of the roller (roller 2), which is kept constant during each individual test.

The summary of the tests and results are shown in Table 6.

Figure 5 shows the creepage curves for a velocity of motor 2 equal to $1000 \mathrm{rpm}$ in dry and wet conditions, while Figure 6 reports the same quantities, but for a velocity of motor 2 equal to $700 \mathrm{rpm}$. All the curves are obtained considering the same loading conditions ( $37 \mathrm{~kg}$ vertically on each wheel, around 10 tons/axle in full scale).

By comparing the curves, it is possible to notice that the peak of the friction force moves towards lower values of creepage, as the reference velocity increases. The effect of the velocity is less important when a reduction of the friction coefficient occurs under wet conditions; in fact, in this case, the peak is less pronounced and the curve has a steady trend after it. In addition, the initial slope of the curves is the same in dry and wet conditions, and these trends are not in accordance with Polach's results for the case of drive dynamics [24].

To understand the behavior, let us consider the linear part of the creepage curve that can be described using Kalker's linear theory, as shown in

$$
F_{x}=G_{a b C} \xi .
$$

In (22), $F_{x}$ is the longitudinal friction force, $G$ is the tangent modulus, $a$ and $b$ are the semiaxes of the contact ellipse, 


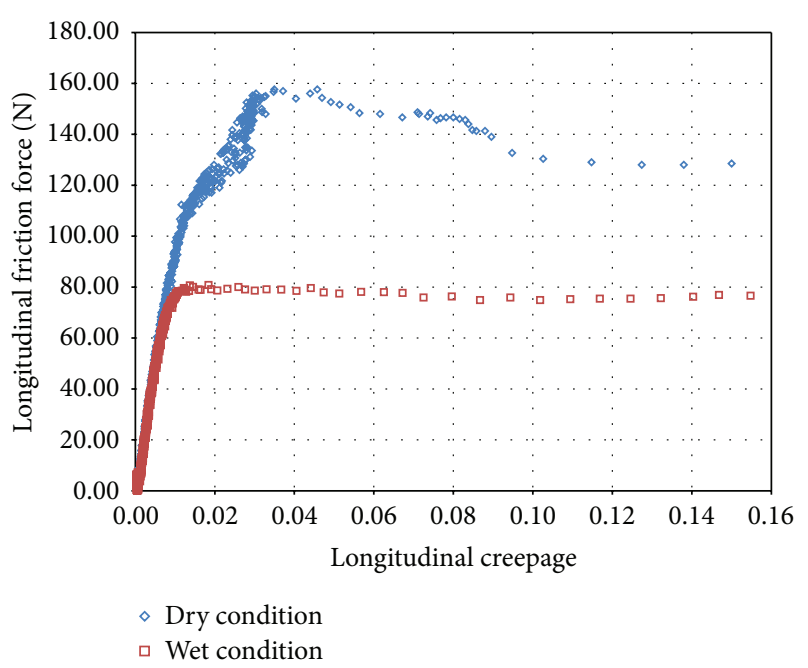

FIGURE 5: Creepage curve in wet and dry conditions considering a reference speed of $1000 \mathrm{rpm}$ and a vertical load of $37 \mathrm{~kg}$ on each wheel.

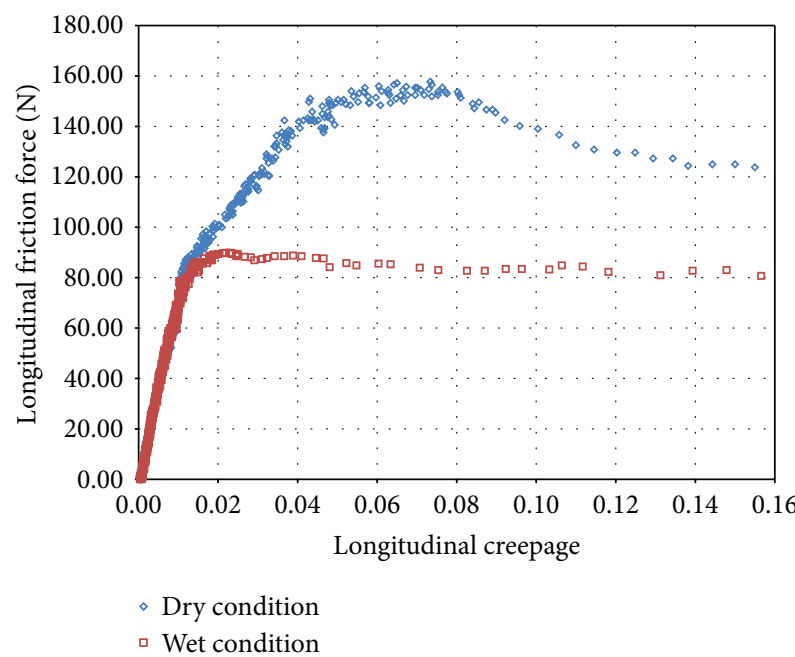

FIGURE 6: Creepage curve in wet and dry conditions considering a reference speed of $700 \mathrm{rpm}$ and a vertical load of $37 \mathrm{~kg}$ on each wheel.

$C_{11}$ is the Kalker's coefficient, which is a function of the ratio of $a, b$ and of the Poisson modulus, and $\xi$ is the longitudinal creepage. Therefore, the slope of the linear part of the creepage curve depends on the elastic characteristics of the materials in contact. The tests show the same trend, in the linear zone, in wet and dry conditions. This can be explained by the two materials in contact being always the same and the water being removed from the contact area, so there is no third-party element (water) in the contact patch.

Figures 7 and 8 show the creepage curves at a reference velocity of $358 \mathrm{rpm}$ and at different vertical loads. In the first case, a load of $42 \mathrm{~kg}$ acts on each wheel and, in the second case, the load is increased to $47 \mathrm{~kg}$. From the results, it emerges that the creepage value, which maximizes the friction force, increases as the normal load on the wheel increases.

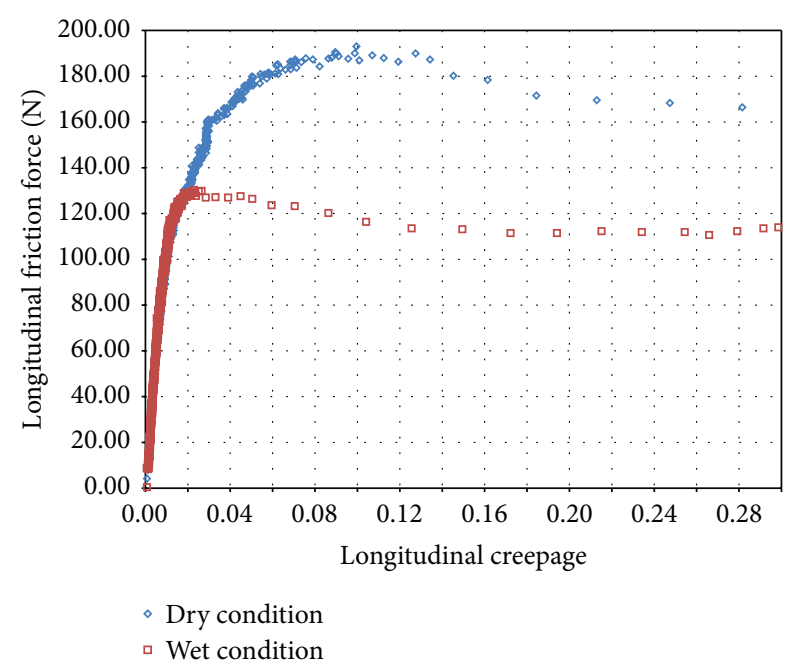

FIgURE 7: Creepage curve in wet and dry conditions considering a reference speed of $358 \mathrm{rpm}$ and a vertical load of $42 \mathrm{~kg}$ on each wheel.

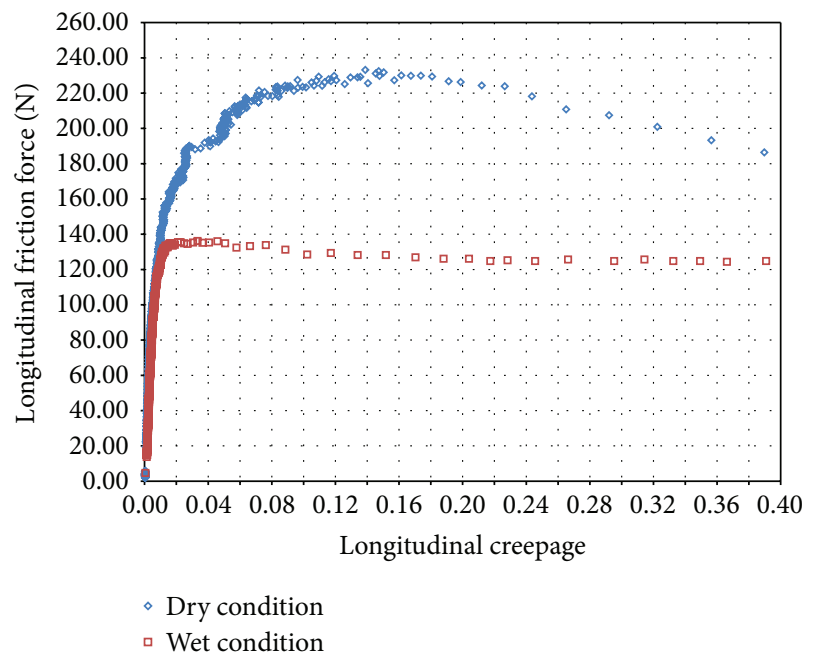

FIGURE 8: Creepage curve in wet and dry conditions considering a reference speed of $358 \mathrm{rpm}$ and a vertical load of $47 \mathrm{~kg}$ on each wheel.

The curves are obtained imposing a torque control on motor 1 and keeping constant the velocity of motor 2 . This strategy has been adopted, since the task in this paper is to characterize the friction properties of the wheel-rail material. Therefore, the first part of the creepage curve has to be obtained with good precision, and this requirement can be achieved only by using a torque control. Furthermore, the method adopted clearly emphasizes the variation of the friction coefficient with the slip velocity, and it is therefore suitable to build a look-up table containing the value of the friction coefficient for different slip velocities.

The RTCONTACT code is essential to obtain all the results shown in this section, because to evaluate the contact force, from the measured torques, it is necessary to know the wheel and the roller rolling radii, which are both affected by the lateral position of the wheelset. 


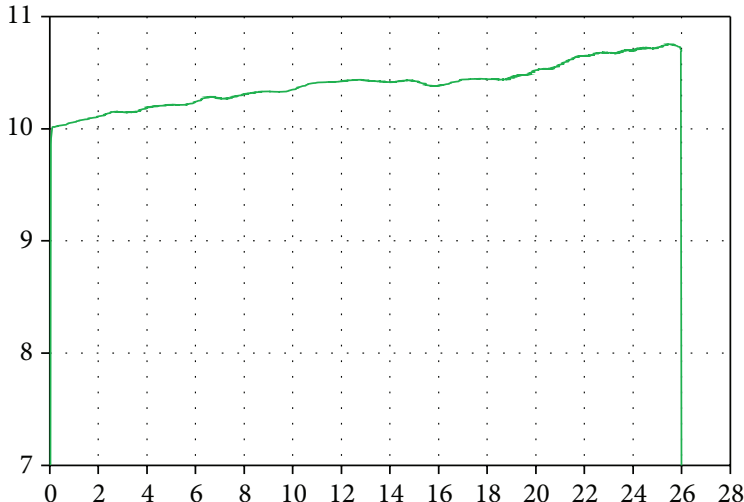

(a) Measured initial profile

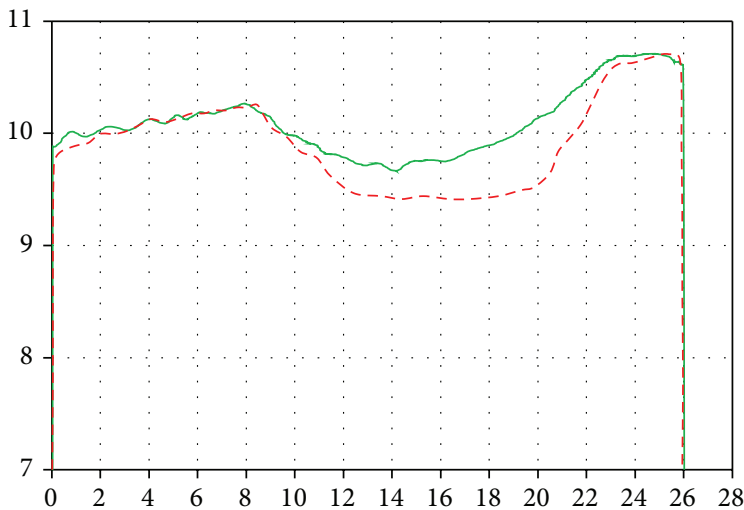

- Measured profile

(c) Profile after the second test $(\xi=0.05)$

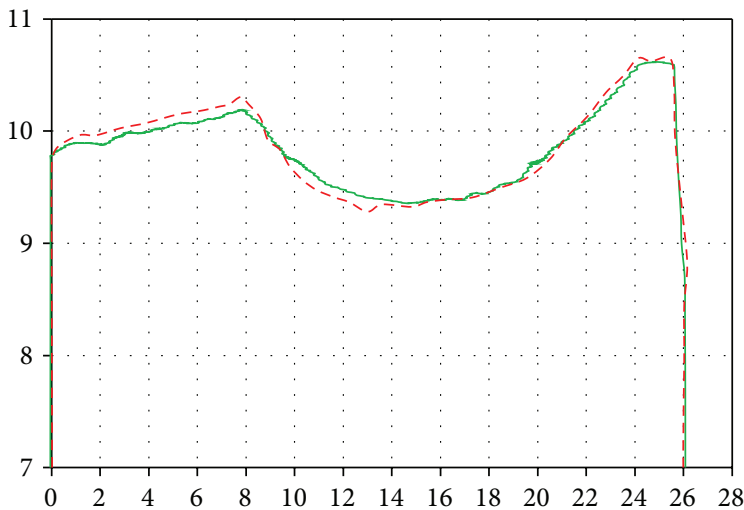

\section{- Measured profile \\ - - - Numerical profile}

(e) Profile after the fourth test $(\xi=0.1)$

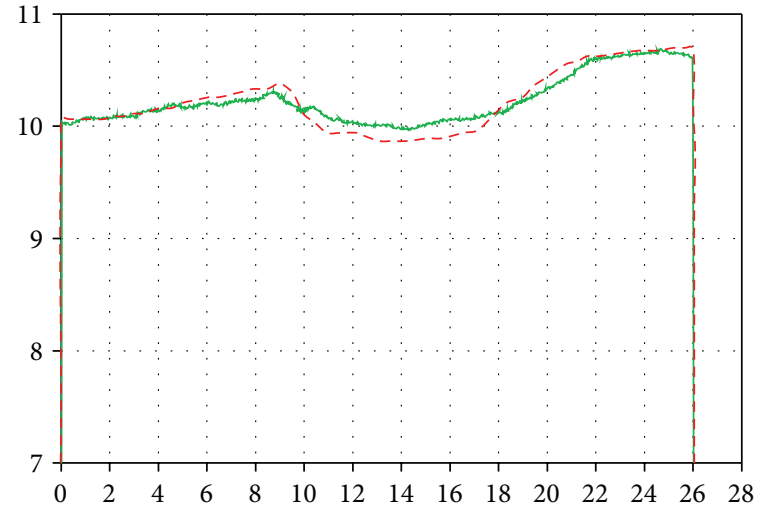

- Measured profile

- - - Numerical profile

(b) Profile after the first test $(\xi=0.25)$

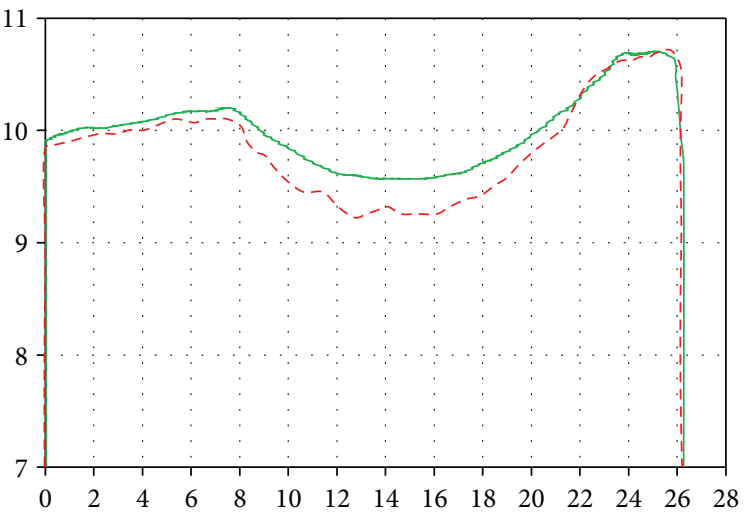

- Measured profile

- - - Numerical profile

(d) Profile after the third test $(\xi=0.05)$

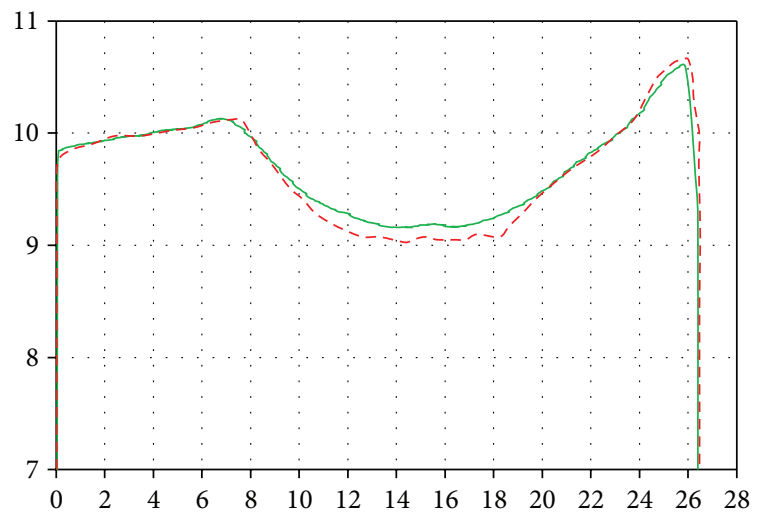

— Measured profile

- - - Numerical profile

(f) Profile after the fifth test $(\xi=0.15)$

FIGURE 9: Shape of the wheel profile after the tests (units in $\mathrm{mm}$ ): comparison of the measured and the numerical results (all the tests are performed with a reference speed of $500 \mathrm{rpm}$ except the last one, performed at $250 \mathrm{rpm}$ ). 


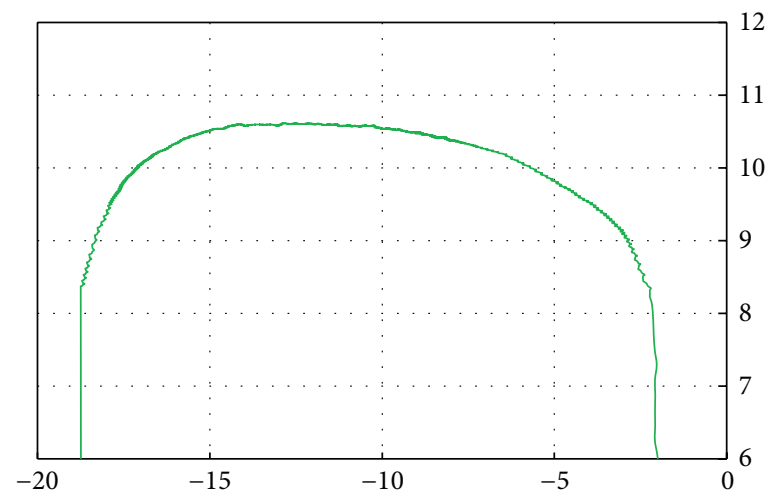

(a) Measured initial profile

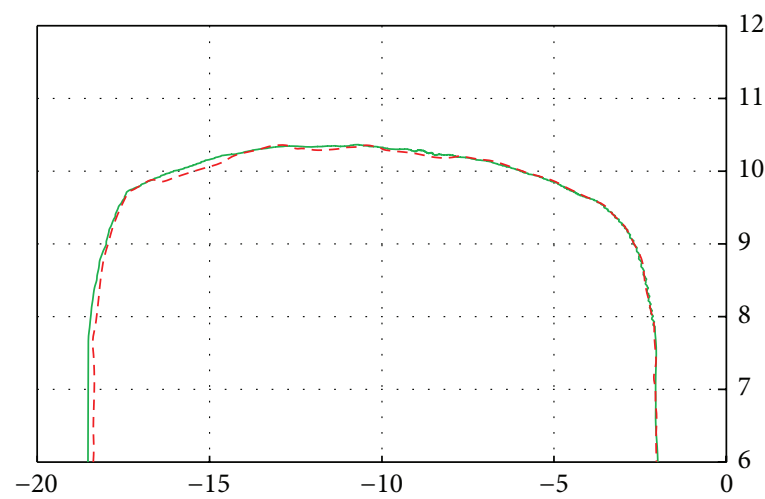

- Measured profile

- - - Numerical profile

(c) Profile after the second test $(\xi=0.05)$

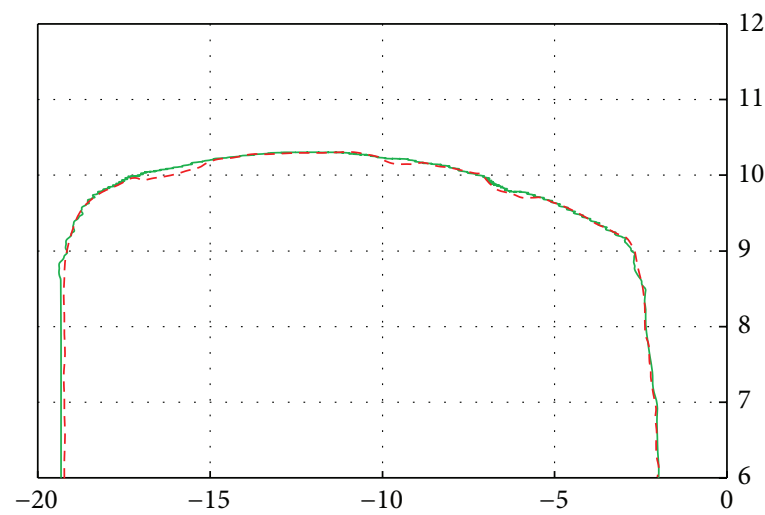

- Measured profile

- - Numerical profile

(e) Profile after the fourth test $(\xi=0.1)$

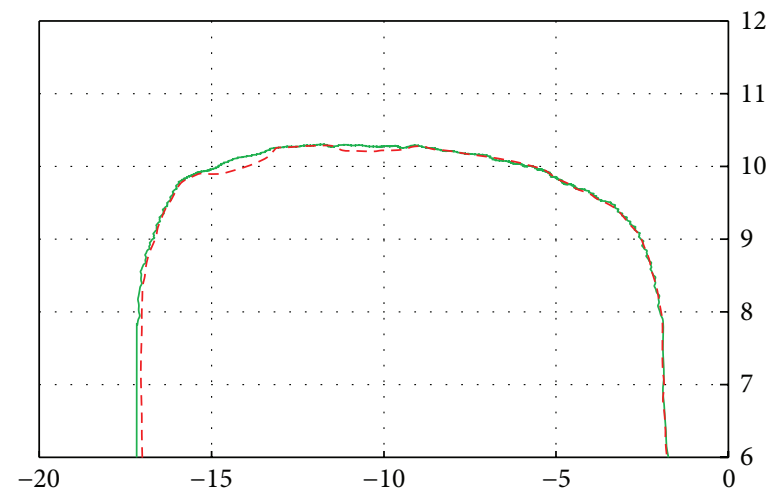

- Measured profile

- - Numerical profile

(b) Profile after the first test $(\xi=0.25)$

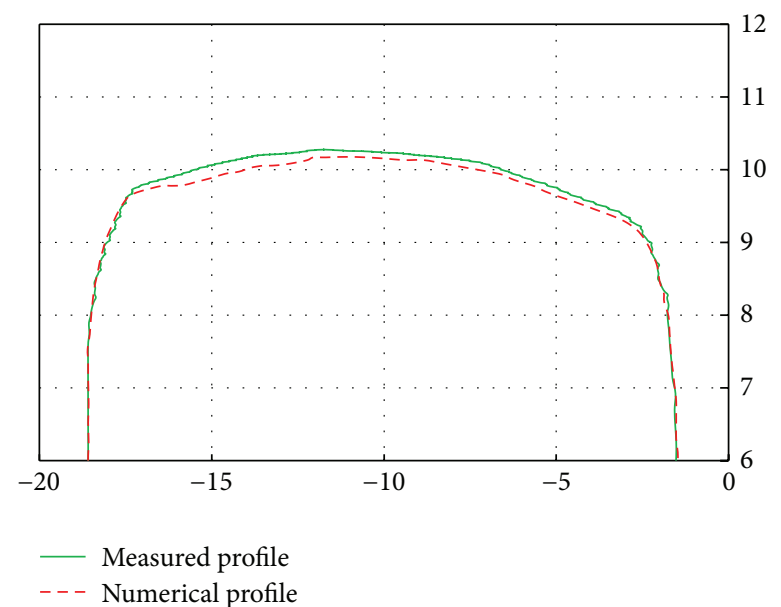

(d) Profile after the third test $(\xi=0.05)$

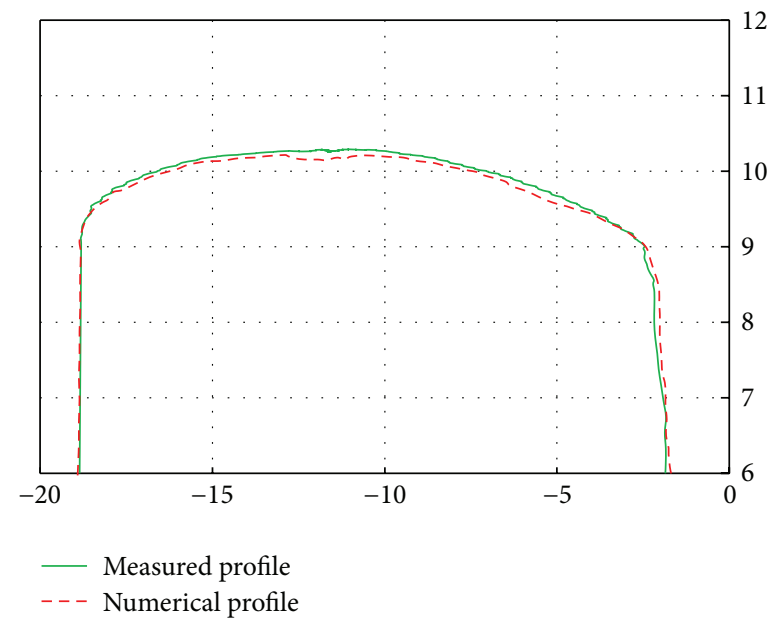

(f) Profile after the fifth test $(\xi=0.15)$

FIGURE 10: Shape of the roller profile after the tests (units in $\mathrm{mm}$ ): comparison of the measured and the numerical results (all the tests are performed with a reference speed of $500 \mathrm{rpm}$ except the last one, performed at $250 \mathrm{rpm}$ ). 


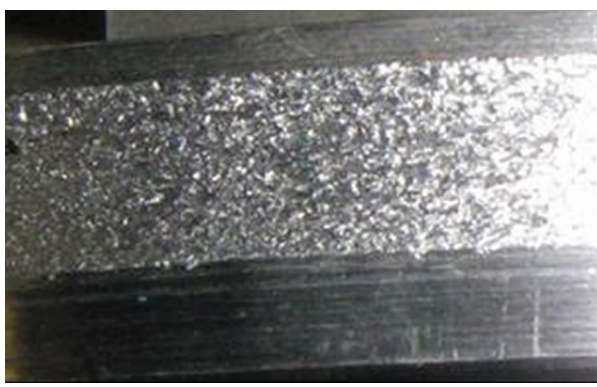

(a) Profile after the first test $(\xi=0.25)$

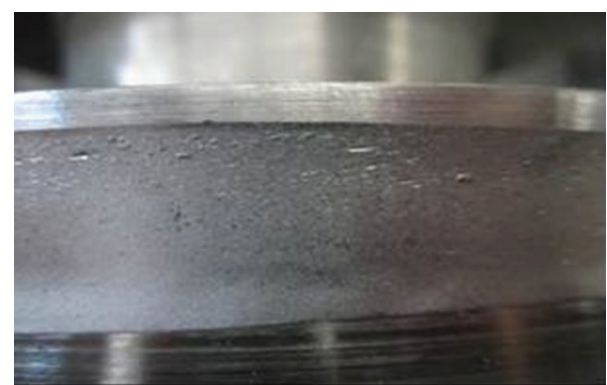

(b) Profile after the third test $(\xi=0.05)$

FIGURE 11: Different wear regimes on the wheel profile.

5.2. Wear. This paragraph shows the results of the wear tests described in Table 5 and compares them with the results of the numerical model. The wear module evaluates the material removed from the wheels and the rollers during the test. The initial profile of the wheel is shown in Figure 9(a), and it is acquired using the laser profilometer. This profile is used by the contact model during the first test as the initial profile. The wear module, in fact, modifies this profile according to the wear rate, calculated on the basis of the measured data during the tests.

Each subsequent test is performed using as its initial profile the last profile measured at the end of the previous test; in this way, it is possible to obtain better agreement between the numerical and the experimental data. The shape of the initial roller profile is shown in Figure 10(a).

Comparing the numerical versus the experimental profile evolution, it can be observed that the results on the rollers are more accurate with respect to the wheelset. The higher differences are found in case of low creepage (0.05), where the numerical model produces higher wear on the wheel profile. Low creepages are the most critical cases to be simulated, because, during the short preliminary tests, when the wear coefficients are estimated, the low creepage produces low wear, and therefore it is difficult to obtain an accurate estimation of the coefficients.

Observing Figure 9, it is possible to notice that a better accordance of the results is reached in the last two tests, and this is due to the fact that the wear coefficients are calibrated during the test.

Additional differences can be found at specific locations, where the numerical model produces local wear that is not found on the experimental tests.

At first it must be observed that the use of softer material produces very high wear rate, equivalent to a very high mileage for a real profile. Therefore any difference is amplified. This is not a problem, but an advantage for a tool, the roller rig, that must be used to test and improve numerical codes. Anyway, to correct the wear model, more tests would be required to improve the wear map and obtain more accurate predictions as those of cases in Figures 9(e) and 9(f).

The tests also allow reproducing different wear regimes that, according to [5], produce a different wear process that can be recognized by analyzing the surface condition after the test.

Since the creepage value has been changed during the wear tests, the wear regime is different in each test, and this causes a different type of wear process on the wheel and the roller. Figure 11(a) shows the surface of the wheel profile after the test performed with $\xi=0.25$, while Figure 11(b) is relative to the test performed with $\xi=0.05$.

\section{Conclusions}

The paper discusses the use of a scaled roller rig to perform adhesion and wear tests with a real-time evaluation of the contact parameters. The roller rig allows control of the creepage between the wheel and the rail, in order to create suitable conditions to evaluate the adhesion and the wear phenomena. The real-time algorithm RTCONTACT is used to calculate the contact force and the material removed from the profiles, directly using the measured data. The algorithm is able to update the wheel and the roller profiles at each time step.

The second section of the paper shows the experimental results as regards the adhesion and the wear tests. The first case reports the adhesion curves in wet and dry conditions for different loads and reference velocities, while the second case shows the shape of the wheel and rail profiles after each test. The adhesion tests are performed using the wheel and the rail profiles directly derived from the standard European profiles S1002 and UIC60. The wear tests are made using simplified wheel profiles and softer materials in order to accelerate the wear process and decrease the time necessary to perform each test. This strategy is adopted since the task of the work is to assess the performance and the accuracy of the wear model and not to characterize the material properties. The work demonstrates how a scaled roller rig can be a good tool to perform the experimental tests and to develop and improve specific contact codes for wear and adhesion studies. The good environmental conditions and the reduced scale allow the integration of all the subsystems in the same platform, in this case working in an RT-environment. In this first activity, the RTCONTACT code, developed by the authors, has been tested and improved, adding specific modules to compute wheel-rail wear. Future development includes the use of profiles with the same material properties used for 
wheels and rails on an actual railroad, in order to refine the material coefficients and obtain results comparable with those obtained on the track.

\section{Conflict of Interests}

The authors declare that there is no conflict of interests regarding the publication of this paper.

\section{References}

[1] N. Bosso and N. Zampieri, "Real-time implementation of a traction control algorithm on a scaled roller rig," Vehicle System Dynamics, vol. 51, no. 4, pp. 517-541, 2013.

[2] U. Olofsson and K. Sundvall, "Influence of leaf, humidity and applied lubrication on friction in the wheel-rail contact: pin-ondisc experiments," Proceedings of the Institution of Mechanical Engineers, vol. 218, no. 3, pp. 235-242, 2004.

[3] J. Sundh, U. Olofsson, and K. Sundvall, "Seizure and wear rate testing of wheel-rail contacts under lubricated conditions using pin-on-disc methodology," Wear, vol. 265, no. 9-10, pp. 14251430,2008

[4] E. A. Gallardo-Hernandez and R. Lewis, "Twin disc assessment of wheel/rail adhesion,” Wear, vol. 265, no. 9-10, pp. 1309-1316, 2008.

[5] P. J. Bolton and P. Clayton, "Rolling-sliding wear damage in rail and tyre steels," Wear, vol. 93, no. 2, pp. 145-165, 1984.

[6] N. Tassini, X. Quost, R. Lewis, R. Dwyer-Joyce, C. Ariaudo, and N. Kuka, "A numerical model of twin disc test arrangement for the evaluation of railway wheel wear prediction methods," Wear, vol. 268, no. 5-6, pp. 660-667, 2010.

[7] A. Kapoor, F. J. Franklin, S. K. Wong, and M. Ishida, "Surface roughness and plastic flow in rail wheel contact," Wear, vol. 253, no. 1-2, pp. 257-264, 2002.

[8] D. Danks and P. Clayton, "Comparison of the wear process for eutectoid rail steels: field and laboratory tests," Wear, vol. 120, no. 2, pp. 233-250, 1987.

[9] R. Nilsson, "Wheel and rail wear-measured profile and hardness changes during 2.5 years for Stockholm commuter traffic," Railway Engineering, 2000.

[10] M.-S. Kim and H.-M. Hur, "Application of braking/traction control systems to the scaled active steering testbed in the railway vehicle," WSEAS Transactions on Systems and Control, vol. 4, no. 7, pp. 296-305, 2009.

[11] K. Nagase, "A study of adhesion between the rails and running wheels on main lines: results of investigations by slipping adhesion test bogie," Proceedings of the Institution of Mechanical Engineers, vol. 203, pp. 33-43, 1989.

[12] F. W. Carter, "On the action of a locomotive driving wheel," Proceedings of Royal Society of London A, vol. 112, pp. 151-157, 1912.

[13] W. Zhang, J. Chen, X. Wu, and X. Jin, "Wheel/rail adhesion and analysis by using full scale roller rig," Wear, vol. 253, no. 1-2, pp. 82-88, 2002.

[14] F. Braghin, S. Bruni, and F. Resta, "Wear of railway wheel profiles: a comparison between experimental results and a mathematical model," Vehicle System Dynamics, vol. 37, pp. 478489, 2003.

[15] A. Jaschinski, H. Chollet, S. Iwnicki, A. Wickens, and J. Von Würzen, "The application of roller rigs to railway vehicle dynamics," Vehicle System Dynamics, vol. 31, no. 5-6, pp. 345392, 1999.

[16] N. Bosso, A. Gugliotta, and A. Somà, "Comparison of different scaling techniques for the dynamics of a bogie on roller rig," Vehicle System Dynamics, vol. 37, pp. 514-530, 2003.

[17] N. Bosso, A. Gugliotta, and A. Somà, "Simulation of narrow gauge railway vehicles and experimental validation by mean of scaled tests on roller rig," Meccanica, vol. 43, no. 2, pp. 211-223, 2008.

[18] N. Bosso, A. Gugliotta, and A. Somà, "Dynamic behavior of a railway wheelset on a roller rig versus tangent track," Shock and Vibration, vol. 11, no. 3-4, pp. 467-492, 2004.

[19] N. Bosso, A. Gugliotta, and N. Zampieri, "RTCONTACT: an efficient wheel-rail contact algorithm for real-time dynamic simulations," in Proceedings of the ASME/ASCE/IEEE Joint Roller Conference (JRC2 '12), pp. 1-10, Philadelphia, Pa, USA, 2012.

[20] J. J. Kalker, Three-Dimensional Elastic Bodies in Rolling Contact, Kluwer Academic Publishers, 1990.

[21] J. J. Kalker, "A fast algorithm for the simplified theory of rolling contact," Vehicle System Dynamics, vol. 11, no. 1, pp. 1-13, 1982.

[22] R. Enblom and M. Berg, "Simulation of railway wheel profile development due to wear influence of disc braking and contact environment," Wear, vol. 258, no. 7-8, pp. 1055-1063, 2005.

[23] R. Lewis and U. Olofsson, "Mapping rail wear regimes and transitions," Wear, vol. 257, no. 7-8, pp. 721-729, 2004.

[24] O. Polach, "Influence of locomotive tractive effort on the forces between wheel and rail," Vehicle System Dynamics, vol. 35, no. 1, pp. 7-22, 2001. 

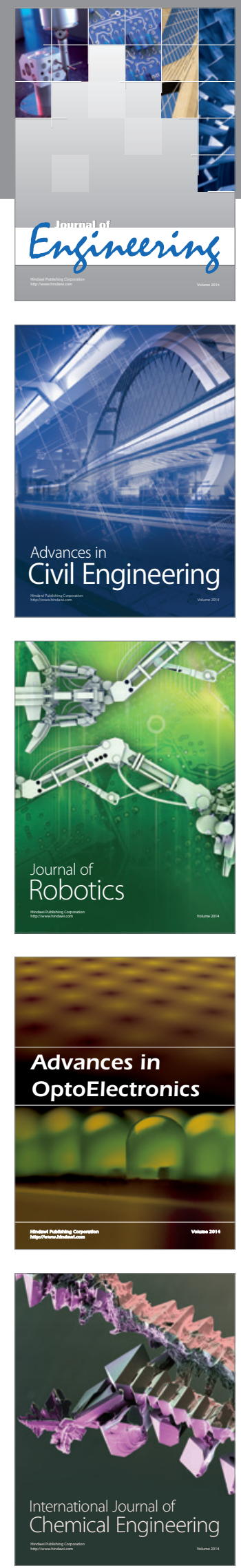

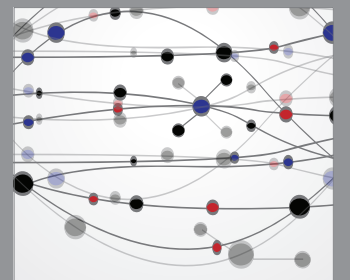

The Scientific World Journal
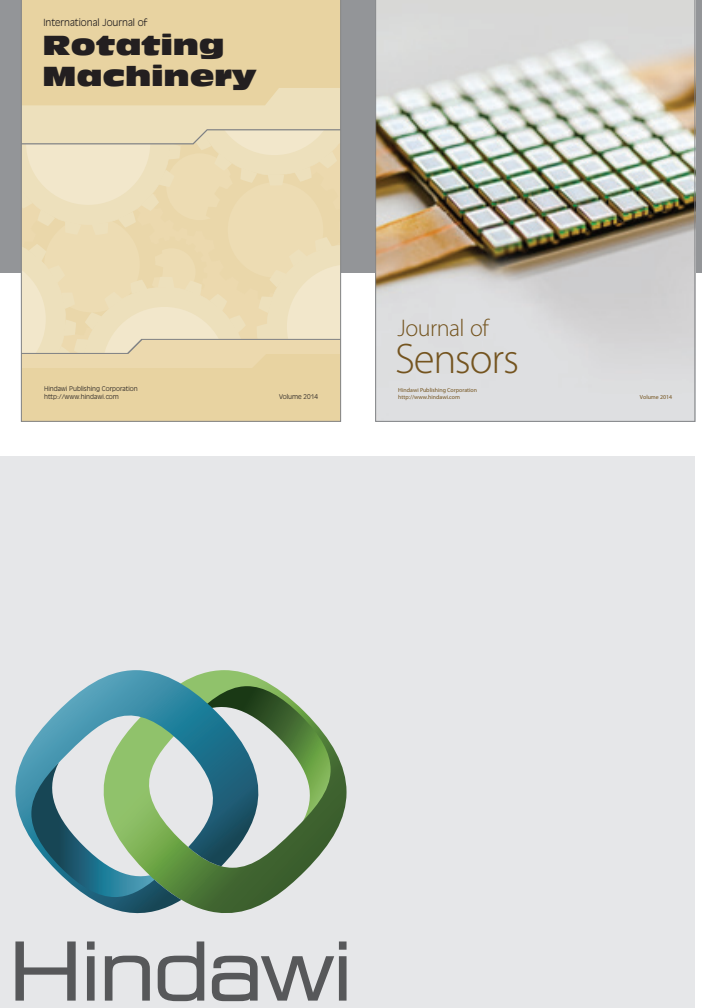

Submit your manuscripts at http://www.hindawi.com
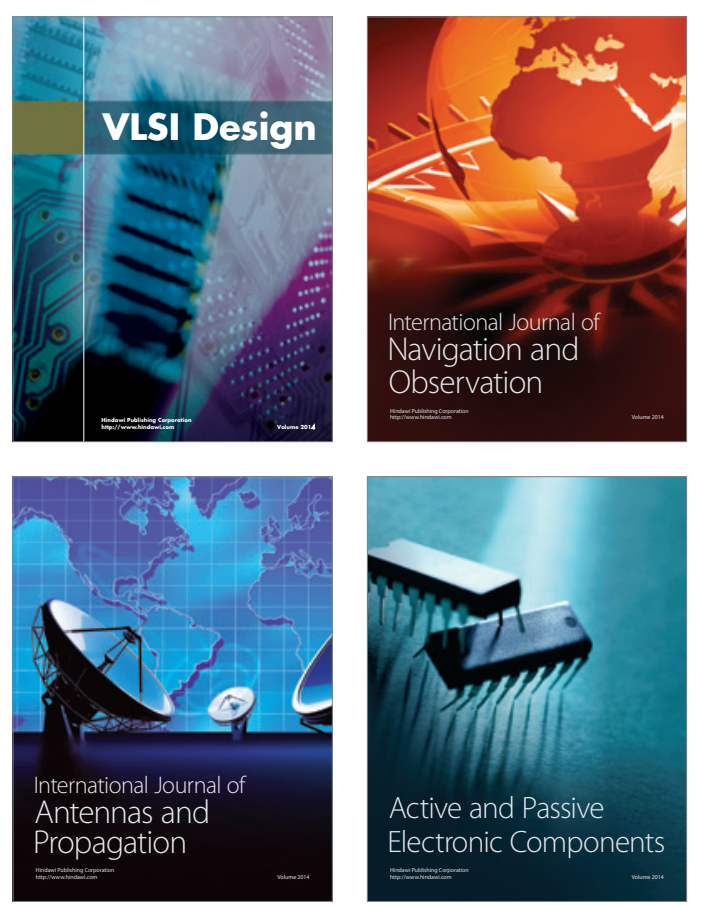
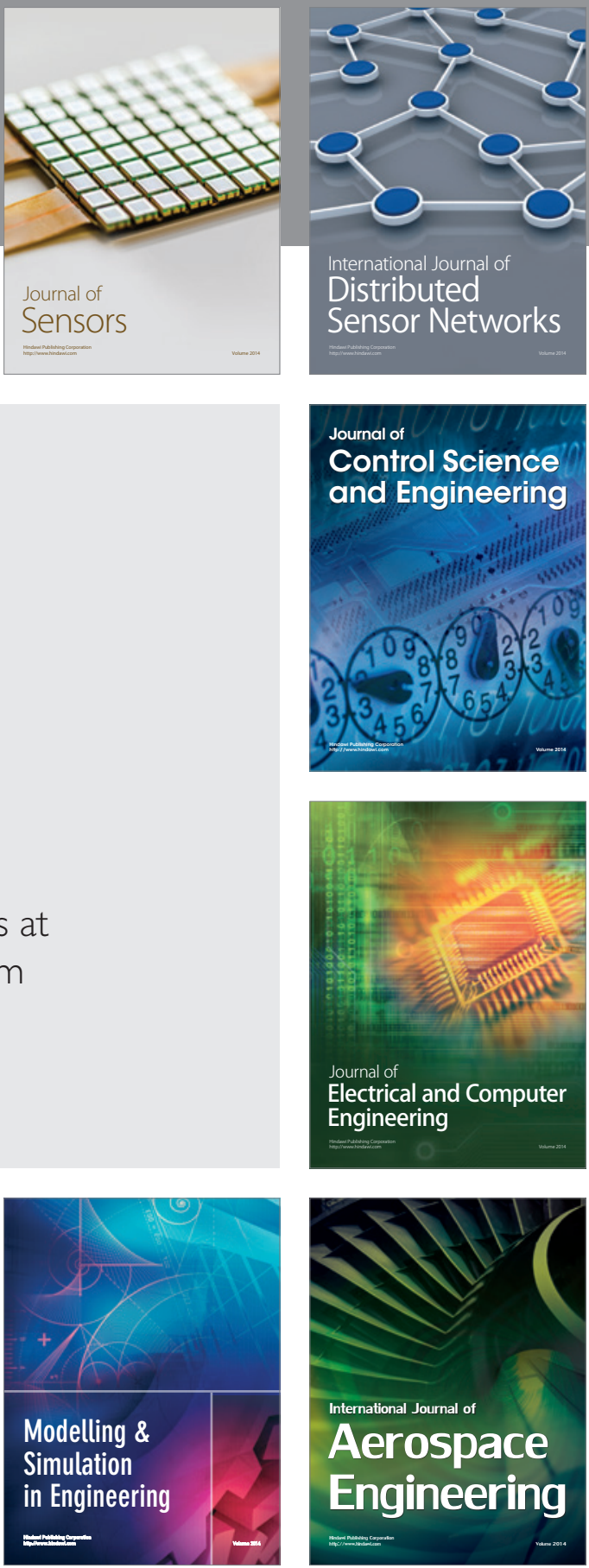

Journal of

Control Science

and Engineering
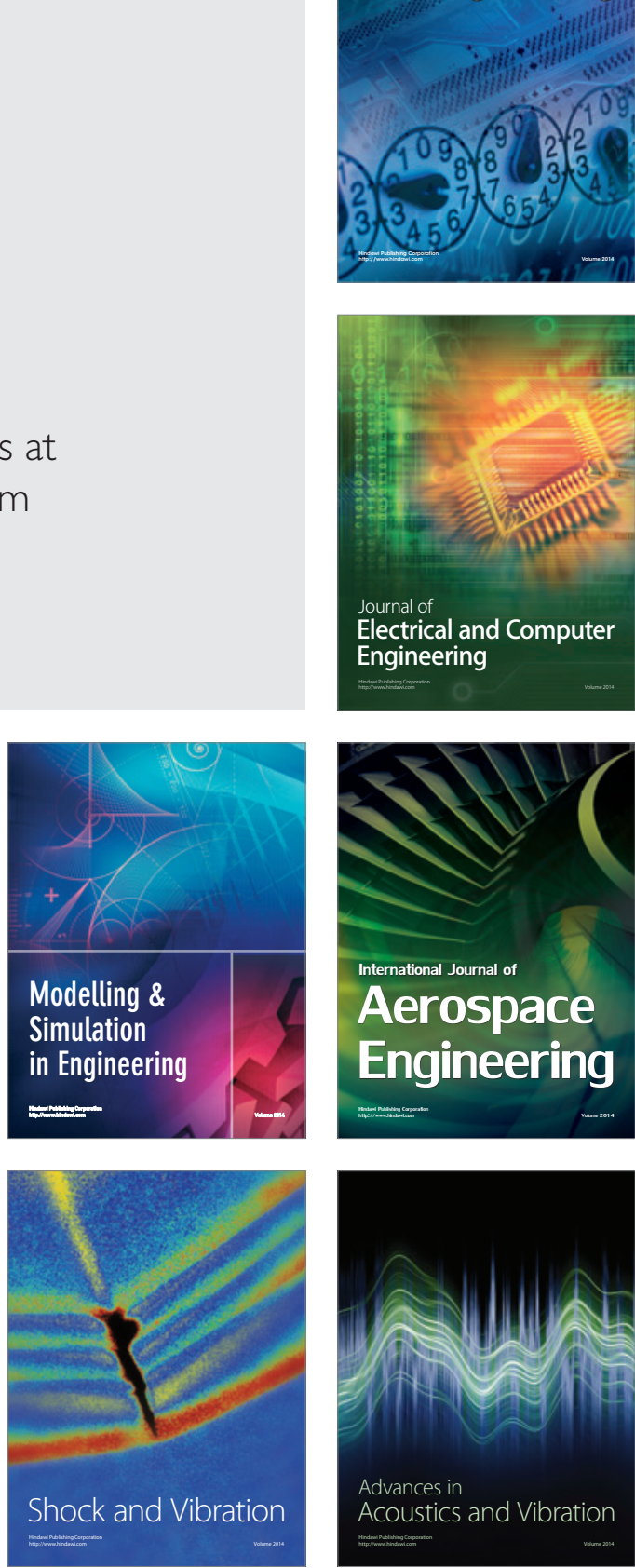